\title{
Editorial
}

\section{Targeted gene repair}

In 1993 and 1994, my laboratory was investigating the role of RNA in extending the half-life of DNA molecules conjoined at homologous regions during the initial phase of recombination known as synapsis. ${ }^{1,2}$ We observed that the length of complementary sequence required for stable hybridization during DNA pairing was reduced when one of the partners contained RNA. Since we believed that DNA pairing was the rate-limiting step of homologous recombination, we reasoned that controlling this phase might improve the frequency of gene targeting in cells from various origins.

Previous work in mammalian cultured cells had demonstrated that gene targeting or gene replacement was a rare event (see Ref. 3 for review) and significant barriers to success had been established by other workers in the field. This information prompted us to formulate the alternative strategy, known as targeted gene repair. In essence, we envisioned repairing a specific mutation rather than replacing an entire dysfunctional gene. Similar strategies using site-directed nucleotide conversion had been attempted in yeast, ${ }^{4}$ but were believed to be target- and context-dependent. In our case, however, a novel chimeric oligonucleotide (Figure 1) containing regions of RNA to enhance binding affinity was designed as a tool to facilitate this process. Conceptually, the oligonucleotide would localize to the target site and form a stable complex. A DNA region containing a single 'incorrect nucleotide' would be placed between the two RNA bridges, producing the mismatched base pair and a distorted helical structure upon binding. The mispaired bases would be recognized by a genetic repair system, which converts the targeted base in the genome using information provided by the chimeric oligonucleotide template.

Initially, we corrected a mutation in episomal copies of the human alkaline phosphatase gene. ${ }^{5}$ Confirmation of genetic nucleotide repair was obtained by direct DNA sequencing without PCR amplification as well as by phenotypic expression. We next corrected the sickle cell mutation with the human $\beta$-globin gene and verified conversion by many criteria, including Southern blot hybridization. ${ }^{6}$ Concern was raised regarding the analytical procedures used to calculate absolute frequency in mammalian gene-targeting experiments. To address this issue, we carried out additional controls ${ }^{7}$ and obtained isogenic clones of corrected cells. These results supported our earlier conclusions.

Although a number of groups have reproduced ${ }^{7}$ and extended $^{8-11}$ the work, there are still questions surround- ing the molecular basis of the technique. Why do some groups have astounding success while others fail to see even the smallest of changes? Clearly, nuclear uptake by the targeted cell is critical and if transfection efficiency is low, conversion events will remain undetectable. Because of length, structure, chemistry and chimeric content of the oligonucleotide, the efficiency of synthesis is low and heterogeneous. Ensuring the purity of the chimeric oligonucleotide is therefore essential. How valid are the reported high frequencies of correction? The most stringent molecular analyses must be used to establish conversion frequencies, including Southern blots, ${ }^{6}$ Western blots ${ }^{10,11}$ and perhaps even enzyme activity assays, ${ }^{9}$ before statements about absolute frequency can be made with confidence. Frequencies of genomic targeting are likely to be cell-specific, but still should be verified by independent assays. For example, in our hands, the newly developed HeLa cell targeting system for the $\beta$-globin gene realizes maximum, validated correction of $2 \%$ routinely and reproducibly using the standard chimera design. Does transcription influence the rate of conversion? Although transcriptional activity can improve the accessibility of a genomic target, we have found that non-transcribed genes such as $\beta$-globin in HeLa cells can be successfully converted (L Peng et al in preparation). Can this technique transfer from cell culture to animal models and then to humans? One of the most significant hurdles to successful genetic therapy has been the transition of cell culture results to animal model applications. For chimeradirected repair, this barrier has already been partially overcome ${ }^{9}$ and further animal trials are underway. Are all mutated genes amenable to correction? Since many genes have been successfully targeted in mammalian, $, 9,11$, bacterial $^{12}$ and plant cells, ${ }^{13}$ there does not appear to be a DNA sequence restriction. On the other hand, not enough genes have been modified to exclude context dependency as a parameter of this approach. How does this technique differ from other gene targeting protocols? Gene knock-out and gene replacement strategies rely on homologous recombination while this technique emphasizes DNA repair. Other oligonucleotide-based strategies utilize single-stranded DNA molecules that can become degraded in the cell or promote random mutageneses or aberrant recombination events. We have detected no untargeted genomic changes and no elevation in recombination frequencies. While single-stranded oligos promote D-loop formation, the chimera forms a double Dloop enabling correction or conversion of both strands of the target (see Figure 1). Finally, this technique can replace/exchange, insert or delete DNA bases using the same molecular structure, a feature unique to this 


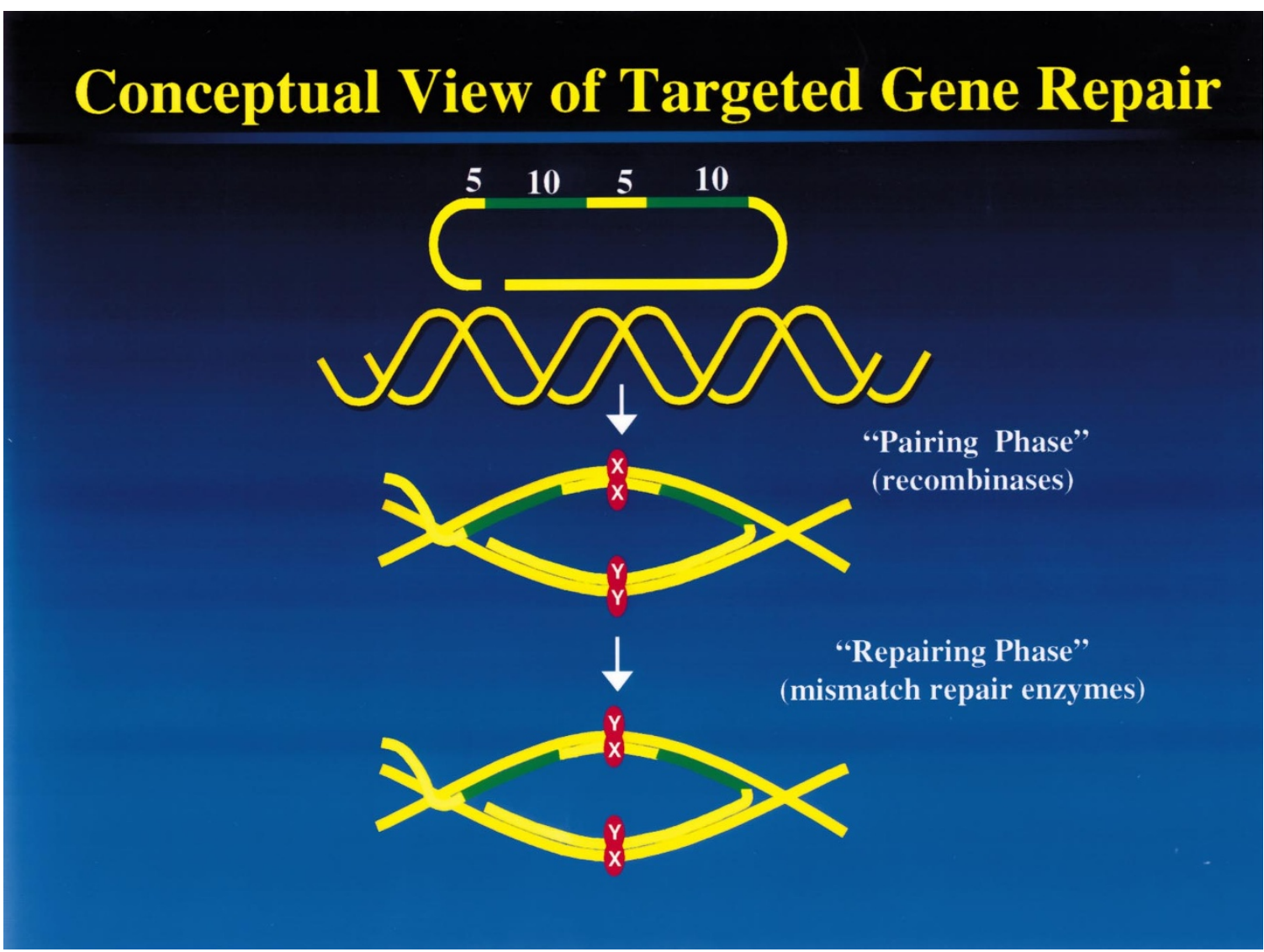

Figure 1 Chimeric oligonucleotide directed gene repair. The chimeric oligonucleotides, in standard form, consist of RNA regions (green) juxtaposed to DNA regions (yellow). The molecule is designed so that a double hairpin configuration forms. The RNA bridges provide more stability after hybridization to the DNA target and the folded ends prevent concatermerization upon entering the cell. The five DNA bases on the top strand and the corresponding complementary bases on the bottom strand are designed to form the mismatched base pairing with the target site. The opening in the chimera enables topological intertwining of strands during the pairing phase. All RNA residues are modified to protect against enzymatic degradation. The four stranded (double D-loop) structure is an important reaction intermediate as it ensures that both strands of the target are converted or corrected.

approach. By what mechanism does chimera-directed gene repair occur? The original idea centered around the use of DNA repair to mediate the change and recent work in my laboratory has focused around proving or disproving this hypothesis. To this end, we have developed a cellfree system using extracts from mammalian cells to correct a point mutation and a single base deletion. ${ }^{14}$ Successful correction is dependent on the properly designed chimera and proteins involved in the mismatch repair pathway such as MutS. As shown in Figure 1, the data support the notion that the chimera binds to the DNA and forms a double D-loop structure with both strands of the chimera hybridizing to both strands of the target. In the second step, mismatch repair enzymes identify, remove and replace the targeted nucleotides using the sequence information provided by the chimera as a template. This mammalian cell-free system, and a related bacterial system ${ }^{12}$ enable direct testing of this model by biochemical and genetic means. Mechanistic studies can be translated into cell-based experiments as has recently been seen in the generation of isogenic clones and the demonstration of sustained inheritance. ${ }^{15}$

Novel genetic therapies must be evaluated without emotion, enthusiasm or commercial pressure. Results must initially be 'understated' rather than 'overstated' and within the context of an ever-expanding universe of hope, vigorous retesting of the data, using the most stringent assay systems available, is scientifically prudent. Even with these concerns however chimera-directed gene repair is likely to have an impact on gene therapy of many inherited and acquired diseases in the near future. It may also prove to be a useful and important research tool, for disciplines such as functional genomics and pharmacogenetics.

EB Kmiec Department of Microbiology Thomas Jefferson University 700 Butler Avenue Doylestown, PA USA

\section{References}

1 Kotani H, Kmiec EB. Transcription activates RecA-promoted homologous pairing of nucleosomal DNA. Mol Cell Biol 1994; 14: 1949-1955.

2 Kotani H, Kmiec EB. A role for RNA synthesis in homologous pairing events. Mol Cell Biol 1994; 14: 6097-6106.

3 Yanez RJ, Porter AG. Therapeutic gene targeting (review). Gene Therapy 1998; 5: 149-159.

4 Moerschell RP, Tsunasawa S, Sherman F. Transformation of 
yeast with synthetic oligonucleotides. Proc Natl Acad Sci USA 1988; 85: 524-528.

5 Yoon K, Cole-Strauss A, Kmiec EB. Targeted gene correction of episomal DNA in mammalian cells mediated by a chimeric RNA-DNA oligonucleotide. Proc Natl Acad Sci USA 1996; 93: 2071-2076.

6 Cole-Strauss A et al. Correction of the mutation responsible for sickle cell anemia by an RNA/DNA oligonucleotide. Science 1996; 273: 1386-1389.

7 Cole-Strauss A, Nöe A, Kmiec EB. Recombinational repair of genetic mutations. Antisens Nucl Acid Drug Dev 1997; 7: 211-216.

8 Kren BT, Cole-Strauss A, Kmiec EB, Steer CJ. Targeted nucleotide exchange in the alkaline phosphatase gene of HuH-7 cells mediated by a chimeric RNA/DNA oligonucleotide. Hepatology 1997; 25: 1462-1468.

9 Kren BT, Bandyopadhyay P, Steer CJ. In in vivo site-directed mutagenesis of the factor IX gene by chimeric RNA/DNA oligonucleotides. Nature Med 1998; 4: 1-6.

10 Bartlett RJ et al. Targeted revision of the mdx point mutation.
Proceedings from 1998 Miami Nature Biotechnology Winter Symposium. Nature Biology Short Reports 1998; 9: 163-165.

11 Lai LW, O'Connor HM, Lien YH. Correction of carbonic anyhydrase II mutation in renal tubular cells by chimeric RNA/DNA oligonucleotide. Conference Proceedings: 1st Annual Meeting of the American Society of Gene Therapy. American Society of Gene Therapy, 183a.

12 Metz R et al. Mismatch directed repair mechanism for in in vivo targeted mutagenesis in Escherichia coli employing synthetic RNA/DNA oligonucleotides. J Mol Biol 1998 (submitted).

13 Kipp PB, Van Eck JM, Beetham PR, May GD. Gene targeting in plants via site-directed mutagenesis. In: Kmiec EB (ed). Gene Targeting Protocols. Humana Press: Totowa, NJ 1998 (in press).

14 Cole-Strauss A et al. A mammalian cell-free extract that directs chimera RNA/DNA oligonucleotide-mediated gene targeting. Nucleic Acids Res 1998 (submitted).

15 Alexeev V, Yoon K. Stable and inheritable changes in genotype and phenotype of albino melanocytes induced by an RNA-DNA oligonucleotide. Nature Biotech 1998 (in press). 